${ }^{1}$ Divisão de Endocrinologia, Departamento de Clínica Médica, Hospital Universitário Clementino Fraga Filho, Universidade Federal do Rio de Janeiro, Rio de Janeiro, RJ, Brasil ${ }^{2}$ Divisão de Endocrinologia, Departamento de Clínica Médica, Hospital Federal de Bonsucesso, Rio de Janeiro, RJ, Brasil ${ }^{3}$ Hospital das Clínicas, Faculdade de Medicina da Universidade de São Paulo, São Paulo, SP, Brasil

${ }^{4}$ Unidade de Endocrinologia e Metabologia (SEMPR), Universidade Federal do Paraná, Curitiba, PR, Brasi ${ }^{5}$ Hospital Municipal Souza Aguiar,

Secretaria Municipal de Saúde do Rio de Janeiro, Rio de Janeiro, RJ, Brasil

${ }^{6}$ Disciplina de Endocrinologia, Escola Paulista de Medicina, Universidade

Federal de São Paulo, São Paulo, SP, Brasi

${ }^{7}$ Departamento de Patologia e

Diagnóstico Oral, Faculdade de

Odontologia, Universidade Federal do Rio de Janeiro, Rio de Janeiro, RJ, Brasil

${ }^{8}$ Divisão de Reumatologia, Escola Paulista de Medicina, Universidade Federal de São Paulo, São Paulo, SP, Brasil ${ }^{9}$ Departamento de Medicina

Clínica, Universidade Federal do

Ceará, Fortaleza, CE, Brasil

${ }^{10}$ Faculdade de Medicina, Centro

Universitário de Belo Horizonte.

Belo Horizonte, MG, Brasil

${ }^{11}$ Divisão de Endocrinologia, Hospital

Felício Rocho, Belo Horizonte, MG, Brasil

${ }^{12}$ Divisão de Endocrinologia,

Santa Casa de Belo Horizonte,

Belo Horizonte, MG, Brasil

${ }^{13}$ Laboratório de Endocrinologia Celular

e Molecular (LIM-25) e Unidade de

Doenças Osteometabólicas, Divisão de

Endocrinologia, Hospital das Clínicas,

Faculdade de Medicina, Universidade de São Paulo, São Paulo, SP, Brasil

${ }^{14}$ Divisão de Endocrinologia e Diabetes Faculdade de Medicina da Universidade de Pernambuco, Recife, PE, Brasil

Correspondence to:

Miguel Madeira

Hospital Universitário Clementino

Fraga Filho - Universidade Federal do Rio de Janeiro

Rua Prof. Rodolpho Paulo Rocco, 255

$9^{\circ}$ andar (Serviço de Endocrinologia)

Ilha do Fundão

21941-590 - Rio de Janeiro, RJ, Brasi

migmadeira@gmail.com

Received on Apr/4/2020

Accepted on July/13/2020

DOI: 10.20945/2359-3997000000301

\section{Prevention and treatment of oral adverse effects of antiresorptive medications for osteoporosis - A position paper of the Brazilian Society of Endocrinology and Metabolism (SBEM), Brazilian Society of Stomatology and Oral Pathology (Sobep), and Brazilian Association for Bone Evaluation and Osteometabolism (Abrasso)}

Miguel Madeira ${ }^{1,2}$

https//orcid.org/0000-0001-6752-2880

André Caroli Rocha ${ }^{3}$

https://orcid.org/000-0003-0070-0640

Carolina Aguiar Moreira 4

https://orcid.org/0000-0002-9908-4907

Águida Maria Menezes Aguiar 5

https://orcid.org/0000-0002-9666-9272

Sergio Setsuo Maeda 6

https://orcid.org/ 0000-0002-2669-4245

Abel Silveira Cardoso ${ }^{7}$

https://orcid.org/0000-0001-9771-3960

Charlles Heldan de Moura Castro ${ }^{8}$

https://orcid.org/0000-0001-9144-996X

Catarina Brasil D'Alva ${ }^{9}$

https://orcid.org/0000-0001-7332-984

Barbara Campolina Carvalho Silva ${ }^{10,11,12}$

https://orcid.org/0000-0001-7276-581X

Bruno Ferraz-de-Souza ${ }^{13}$

https://orcid org/0000-0003-4863-2544

Marise Lazaretti-Castro 6

https://orcid.org/ 0000-0001-9186-2834

Francisco Bandeira ${ }^{14}$

https://orcid.org/0000-0003-0290-0742

Sandra R. Torres ${ }^{7}$

https://orcid.org/0000-0002-9271-3707

\section{ABSTRACT}

Antiresorptive therapy is the main form of prevention of osteoporotic or fragility fractures. Medicationrelated osteonecrosis of the jaw (MRONJ) is a relatively rare but severe adverse reaction to antiresorptive and antiangiogenic drugs. Physicians and dentists caring for patients taking these drugs and requiring invasive procedures face a difficult decision because of the potential risk of MRONJ. The aim of this study was to discuss the risk factors for the development of MRONJ and prevention of this complication in patients with osteoporosis taking antiresorptive drugs and requiring invasive dental treatment. For this goal, a task force with representatives from three professional associations was appointed to review the pertinent literature and discuss systemic and local risk factors, prevention of MRONJ in patients with osteoporosis, and management of established MRONJ. Although scarce evidence links the use of antiresorptive agents in the context of osteoporosis to the development of MRONJ, these agents are considered a risk factor for this complication. Despite the rare reports of MRONJ in patients with osteoporosis, the severity of symptoms and impact of MRONJ in the patients' quality of life make it imperative for health care professionals to consider this complication when planning invasive dental procedures. Arch Endocrinol Metab. 2020;64(6):664-72

Keywords

Osteoporosis; bisphosphonate; medication-related osteonecrosis of the jaw; antifracture therapy; dental care 


\section{INTRODUCTION}

$\mathrm{T}$ he management of patients with osteoporosis taking antiresorptive drugs is challenging. While antiresorptive drugs are effective and well established in preventing osteoporotic fractures, dentists face difficult decisions when patients taking antiresorptive drugs require invasive dental treatment, mainly due to the risk of medication-related osteonecrosis of the jaw (MRONJ), a rare but severe adverse reaction associated with antiresorptive and antiangiogenic agents (1-4). Interprofessional collaboration is paramount in establishing strategies for improving clinical practice and better assisting these patients. Therefore, a need has arisen for a position paper involving health care professionals who assist patients with osteoporosis treated with antiresorptive drugs requiring dental care. In recognizing the close relationship between osteoporosis treatment and advanced dental care, a task force with representation from the Sociedade Brasileira de Endocrinologia e Metabologia (SBEM; Brazilian Society of Endocrinology and Metabolism), Sociedade Brasileira de Estomatologia e Patologia Oral (Sobep; Brazilian Society of Stomatology and Oral Pathology), and Associação Brasileira de Avaliação Óssea $e$ Osteometabolismo (Abrasso; Brazilian Association for Bone Evaluation and Osteometabolism) convened to perform a comprehensive review of available evidence and publish a joint consensus document on the issue providing up-to-date recommendations for clinical practice.

Osteoporosis is a highly prevalent disease resulting in fragility fractures that reduce quality of life and increase the risk of death. This condition affects mainly postmenopausal women but may also affect older men. Osteoporosis may be secondary to several chronic diseases, including inflammatory disorders (rheumatic, intestinal, and respiratory), malignant conditions (multiple myeloma), endocrine diseases (hypogonadism, primary hyperparathyroidism, and Cushing's syndrome), and conditions that cause intestinal malabsorption $(3,5)$. Medications can also cause osteoporosis, including glucocorticoids, which are the most common cause of secondary osteoporosis (3). In Brazil, available data confirm that osteoporosis incurs in significant individual and social burden: approximately $15 \%$ of female and $13 \%$ of male Brazilian adults present fragility fractures (6), and the annual costs for treatment of osteoporotic fractures may amount to 310 million US dollars (7).
The American Association of Oral and Maxillofacial Surgeons (AAOMS) defines MRONJ as "The presence of exposed jaw bone, or bone that can be probed through an intraoral or extraoral fistula, for at least 8 weeks, in a patient with a history of antiresorptive and/or antiangiogenic therapy, and in the absence of previous radiation therapy to the head and neck" (8). Complications that might be expected in patients with MRONJ are local infection, pain, loss of function, and poor quality of life (3).

Although rare and more commonly seen in patients with cancer, MRONJ is associated with substantial morbidity that can cause anguish to patients and health care professionals, making therapeutic decisions difficult. Therefore, identifying patients at risk of MRONJ and the factors that may predict the development of this complication is extremely important. Here, we discuss the risk of MRONJ and preventive strategies in patients with osteoporosis receiving antiresorptive therapy and undergoing invasive dental procedures.

\section{MATERIALS AND METHODS}

An electronic search of the literature was performed to identify published studies that reported MRONJ and other adverse effects of antiresorptive therapy in patients with osteoporosis from January 2002 to September 2019. Clinical and epidemiological studies reporting on the risk and prevention of MRONJ in patients with osteoporosis in the English language were eligible for the analysis. Reviews, letters to the editors, studies that did not disclose the type of antiresorptive therapy, animal studies, and those not available in the full version were not included.

A multidisciplinary task force was convened including representatives of three Brazilian professional associations with interest in bone metabolism and oral care: the Sociedade Brasileira de Endocrinologia e Metabologia (SBEM; Brazilian Society of Endocrinology and Metabolism), Sociedade Brasileira de Estomatologia e Patologia Oral (Sobep; Brazilian Society of Stomatology and Oral Pathology), and Associação Brasileira de Avaliação Óssea e Osteometabolismo (Abrasso; Brazilian Association for Bone Evaluation and Osteometabolism). Specific questions were proposed and addressed by members of the task force related to the literature review. A draft position was reviewed by coordinators of the three societies, and the task force unanimously approved the final report. 


\section{Osteoporosis treatment and adverse effects}

Approved therapeutic approaches to prevent and treat osteoporosis modify bone remodeling by either slowing the rate of bone loss (antiresorptive) or increasing the rate of bone formation (9). A variety of antiresorptive agents are available, acting through different mechanisms to reduce bone loss. Therefore, the therapeutic choice is guided by pharmacologic characteristics, desired antiresorptive properties, potential benefits, adverse effects, and context-dependent availability. Based on the aim of this paper, only bisphosphonates and denosumab will be discussed, as these antiresorptive agents have been previously associated with MRONJ.

Bisphosphonates are the most common medications used for osteoporosis treatment worldwide, and alendronate, risedronate, ibandronate, and zoledronic acid are the four available molecules approved for this purpose. Bisphosphonates have a very high affinity for the bone mineral matrix, where they deposit and are taken up by osteoclasts during active bone resorption (5). The osteoclast cytoskeleton is disarranged by bisphosphonates, leading to cell apoptosis and inhibition of bone resorption and remodeling.

Besides MRONJ, other adverse effects have been associated to bisphosphonates. Esophageal and gastric dyspeptic symptoms are the most common adverse effects of these agents, but atypical femoral fractures and atrial fibrillation have also been reported $(10,11)$. Since the skeleton can retain bisphosphonates for up to 10 years, depending on the bone turnover rate, the effects of these agents last for months or even years after discontinuation of these drugs (12). This long half-life allows for a more convenient therapeutic dosage, including weekly, monthly, quarterly, or annual regimens, along with periodic drug holidays in low-risk patients (5).

Denosumab is a human monoclonal antibody directed against the receptor activator of nuclear factor- $\kappa \mathrm{B}$ ligand (RANKL), thereby inhibiting the differentiation and activity of osteoclasts and greatly suppressing bone resorption (13). Denosumab is administered as a single subcutaneous injection every 6 months. Unlike bisphosphonates, denosumab does not accumulate in the skeleton and its effects cease immediately upon treatment discontinuation (13). A rebound effect may be observed, with an increased risk of fragility fractures within 12 months from the suspension of this drug (14).

\section{MRONJ epidemiology in patients with osteoporosis}

The prevalence of MRONJ in patients receiving oral bisphosphonates for the treatment of osteoporosis ranges from $0-0.04 \%$, with most reports citing prevalences below $0.001 \%$ (15). A total of 1.04-69 cases of MRONJ per 100,000 patients treated with oral bisphosphonates are described yearly. In clinical trials, the risk for MRONJ among patients treated with either zoledronic acid $(0.017 \%)$ or denosumab $(0.04 \%)$ is comparable to the risk observed in patients assigned to placebo $(0-0.02 \%)$. Thus, based on the current literature, the risk of developing MRONJ among patients with osteoporosis exposed to oral or intravenous bisphosphonates or denosumab is real but remains very low and almost the same or slightly higher than the risk $(0.001 \%)$ in the general population (16). The duration of antiresorptive therapy has also been demonstrated as a risk factor for the development of MRONJ (8). Even though the causes of secondary osteoporosis, such as the use of glucocorticoids, can pose additional risks for MRONJ, this complication can occur in both primary and secondary osteoporosis (17).

A recent Brazilian cross-sectional study evaluated 153 patients taking oral or intravenous bisphosphonate for osteoporosis treatment and 134 individuals using bisphosphonates for metastatic breast cancer. A 3\% prevalence of MRONJ in women with metastatic breast cancer receiving bisphosphonates was identified, while no case of MRONJ was observed in patients receiving these agents for osteoporosis (18).

Reliable epidemiological evidence on the incidence of MRONJ is particularly challenging to obtain due to several limitations regarding sample sizing (generally small), study design (retrospective rather than prospective), study duration, and lack of active casefinding strategies. Underreporting of MRONJ may also lead to inaccurate incidence rates.

\section{Influence of systemic factors on MRONJ development}

Several clinical factors other than antiresorptive therapy have been associated with MRONJ, including diabetes mellitus, rheumatoid arthritis, hypertension, smoking, and use of other medications such as glucocorticoids, antithrombotic agents, immunosuppressants, and proton pump inhibitors $(19,20)$ (Table 1). 
Table 1. Main risk factors for medication-related osteonecrosis of the jaw

\begin{tabular}{l}
\hline Systemic risk factors \\
\hline Diabetes mellitus \\
Rheumatoid arthritis \\
Hypertension \\
Smoking \\
Medications: \\
Antiresorptive agents \\
Antiangiogenic agents \\
Glucocorticoids \\
Antithrombotic agents \\
Immunosuppressants \\
Proton pump inhibitors \\
\hline Local risk factors \\
\hline Dental infection \\
Trauma \\
Invasive oral procedures
\end{tabular}

Long-term use of bisphosphonates and better adherence to treatment may also increase the risk of MRONJ $(15,16)$. A Korean study of patients with osteoporosis reported a significant association between the use of bisphosphonates and the risk of MRONJ, with a dose-response relationship and a higher risk among patients exposed to bisphosphonate therapy for 1.5-2 years compared with those exposed for 0-1.5 year (21). A longitudinal cohort of 61,990 users of alendronate followed up for a mean period of 6.8 years identified 107 cases of MRONJ and showed that recent compared with past use of alendronate was associated with an adjusted odds ratio of 4.13 (95\% confidence interval [CI] 1.94-8.79) (20). The risk of MRONJ was two to three times increased in adherent users with a medication possession ratio greater than $50 \%$. Finally, patients on long-term use of alendronate ( $>5$ years) showed a higher risk of MRONJ than those on shortterm use of this drug (20).

\section{Influence of local factors in the development of MRONJ}

The maxillary bones are highly susceptible to MRONJ since bisphosphonates accumulate mainly in skeletal sites with high bone remodeling activity and because the thin oral mucosa can be easily traumatized $(17,18)$. MRONJ affects the mandible more frequently than the maxilla and is more common in some areas, such as bony prominences, tori, and mylohyoid line (22).
Inflammatory oral disease, such as active periodontal conditions, caries, acute or chronic pulpoperiapical processes, trauma from removable prosthesis or other physical trauma, and poor oral hygiene, are considered local risk factors for MRONJ (23). The mouth is colonized by a large number of bacteria, and the jaw bones are often involved in infectious processes of periodontal or pulp origin. When bisphosphonates accumulate in these sites, tissue repair following induced or physiological trauma fails to occur properly, potentially leading to an area of exposed necrotic bone in the oral environment (23).

All patients starting antiresorptive therapy should be referred to dentists for preventive oral procedures and orientation prior to initiating the osteoporosis treatment. Once patients are receiving antiresorptive therapy, oral surgical interventions, especially dental extractions, are considered predisposing factors associated with MRONJ. A growing body of evidence suggests that dental infection, rather than dental extraction per se, might represent the main local risk factor for MRONJ (24). Considering tooth extraction, those performed traumatically or without primary closure of the socket are more related to the development of MRONJ $(25,26)$.

\section{Drug holiday for MRONJ prevention}

Based on the residual effects of bisphosphonates after long-term therapy, patients at low fracture risk after 5 years of alendronate or 3 years of zoledronic acid can undergo a drug holiday with periodic reassessment. This recommendation aims to minimize safety concerns (27). However, a retrospective population study in a Korean database showed a higher prevalence of MRONJ in the first 3 years after bisphosphonate suspension (28). Although patients with and without cancer were studied, no correlation between dosing frequency or time since bisphosphonate discontinuation and the occurrence of MRONJ was observed in either group (28).

The risk of developing MRONJ is small compared with the antifracture benefits of bisphosphonates in individuals with moderate to high fracture risk using these drugs (29). So far, no evidence-based study has demonstrated efficacy of bisphosphonate drug holiday in preventing MRONJ.

Denosumab has no sustained antiresorptive effect. Hence, drug holiday is not recommended with this 
medication, mainly due to recent concerns about a rapid increase in the risk of vertebral fractures following denosumab discontinuation (30).

Only one study has aimed to evaluate the effect of interrupting oral bisphosphonates before invasive dental procedures for the prevention or treatment of MRONJ (31). In this study, 1,175 patients underwent 2,458 surgical procedures. Some patients underwent the procedure after 2 or 3 months of bisphosphonate withdrawal, and no significant difference in the frequency of MRONJ occurred between patients who did or did not discontinue the medication before tooth extraction.

There is no evidence supporting the suspension of antiresorptive therapy for osteoporosis before dental procedures for preventing MRONJ (31). Position papers from several professional societies and task forces do not include a recommendation for routine discontinuation of bisphosphonate therapy for osteoporosis before invasive dental procedures $(15,16,24,27)$. Additionally, a multicenter retrospective study has shown that the suspension of bisphosphonates has no benefit on the outcome of patients with osteoporosis who developed MRONJ (32).

\section{Predictive factors for MRONJ}

Several studies have attempted to assess the risk of MRONJ using bone turnover markers (BTMs). Marx and cols. (33), in a retrospective, uncontrolled study of 30 patients, have proposed that $\mathrm{C}$-terminal telopeptide (CTX) levels measured before dental surgery could predict MRONJ. Since then, many other studies have been carried out to investigate the ability of BTMs to predict the occurrence of MRONJ, with conflicting results (34-36).

A recent systematic review and meta-analysis of 18 clinical trials involving 2,301 patients receiving bisphosphonates and scheduled for dental surgery showed no significant difference in mean CTX levels between patients who developed MRONJ and those who did not develop this complication (37). Also, no significant differences occurred in the prevalence of MRONJ between patients with CTX levels lower or higher than $150 \mathrm{pg} / \mathrm{mL}(\mathrm{p}=0.92)$. Based on these data, the authors concluded that the measurement of CTX levels for identifying patients at risk of MRONJ is not justified by evidence, and the proposed CTX cutoff value of $150 \mathrm{pg} / \mathrm{mL}$ is not associated with an increased prevalence of this complication (37). Therefore, current evidence does not support using CTX levels before dental procedures for predicting the risk of MRONJ.

Other serum biomarkers were recently assessed in a systematic review (38) analyzing six biomarkers: the bone turnover biomarkers bone alkaline phosphatase, deoxypyridinoline, N-telopeptides of type I collagen, and osteocalcin; the endocrine biomarker parathyroid hormone; and the angiogenesis marker vascular endothelial growth factor. All biomarkers analyzed failed to predict the risk of MRONJ adequately.

Quantitative trabecular and cortical bone measurements have shown to be potentially useful tools in detecting bone changes caused by bisphosphonates in mixed populations of patients taking oral or intravenous bisphosphonate $(39,40)$. In the studies, which evaluated only patients taking oral bisphosphonates for osteoporosis, radiographic and cone-beam computed tomography measurements were not predictive of MRONJ $(41,42)$.

Qualitative evaluation of the jaw bones, such as mandibular cortical index evaluated on panoramic radiographs, has also been shown to be useful in predicting the development of MRONJ in patients with osteoporosis (43). Sclerosis of trabecular bone and thickening of the lamina dura were considered imaging features consistent with bisphosphonate administration (43).

\section{Tooth extractions in patients with osteoporosis using antiresorptive medication}

Performing a dental extraction procedure on a patient using an antiresorptive agent requires care before, during, and after the procedure. The preoperative procedures involve preparing the oral environment, with supragingival scaling, removal of active carious lesions, and orientation of oral hygiene, whenever indicated (44).

Antibiotic prophylaxis should be initiated prior to tooth extraction, and amoxicillin with or without clavulanate is most commonly indicated for this purpose (45). For patients allergic to penicillin, clindamycin may be prescribed. A systematic review has reported different prophylactic regimens starting 48 to 72 hours before the procedure and maintained for 1 to 3 weeks after the procedure (45). There is no uniform approach applied to all patients receiving bisphosphonates with regard to the length of prophylactic antibiotic treatment before and after tooth extraction, but most authors agree that post-extraction treatment regimens should be 
continued until the surgical site has completely healed (45). Antibiotic therapy should be maintained for a longer period if surgical wound repair is delayed (23).

The surgical technique to be performed should be minimally invasive and should provide primary closure of the dental socket (23). Alveoloplasty may facilitate primary closure of the socket after extraction, but surgeons have full discretion in deciding whether to perform it or not (23). The development of MRONJ seems to be related to cases in which the dental alveolus is not primarily closed in the immediate postoperative period, therefore requiring more healing time (23). Careful postoperative follow-up is required until the healing process is complete, which may take longer than usual (46).

Another important recommendation is the completion of an informed consent form, which should make the patient aware of the low risk of dental extraction for the development of MRONJ, and the importance of complying with the recommendations on the postoperative follow-up (47). Figure 1 shows an example of an informed consent form for patients receiving osteoporosis treatment who will undergo invasive dental treatment.

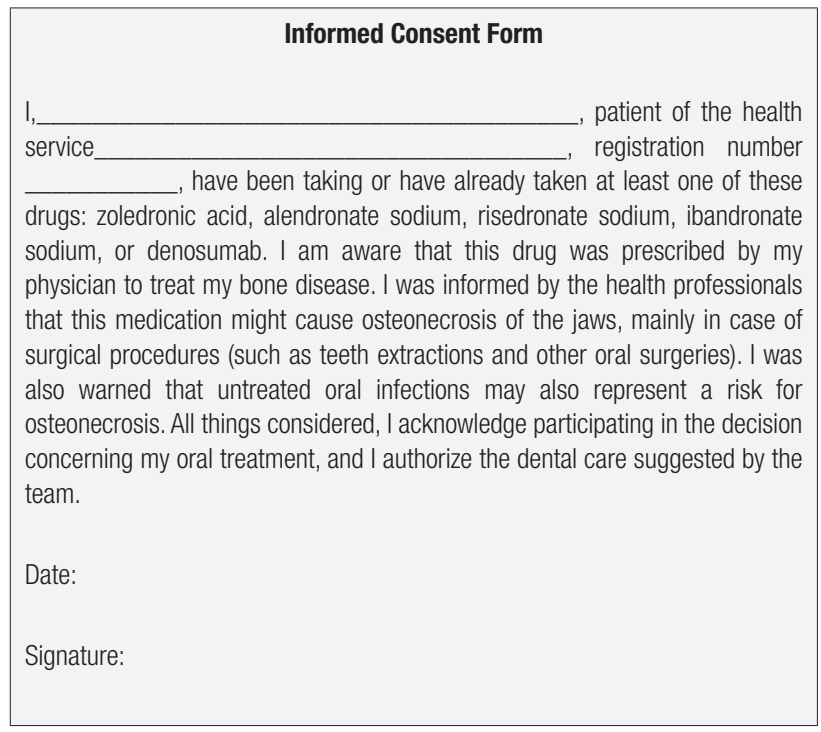

Figure 1. Informed consent form model for patients undergoing osteoporosis treatment who will undergo invasive dental treatment.

\section{Implant placement in patients with osteoporosis undergoing antiresorptive therapy}

The risk of MRONJ after dental implant placement is considered to be comparable to the risk of MRONJ associated with tooth extraction (8). Based on current recommendations, the placement of dental implants should be avoided in patients receiving intravenous antiresorptive or antiangiogenic therapy, whereas treatment with oral antiresorptive drugs is not considered an explicit contraindication for the procedure (48). However, caution is advised in view of conflicting data regarding implant failure and the risk for MRONJ development in patients with osteoporosis.

A study with 235 women with osteoporosis receiving oral bisphosphonates who underwent dental implant placement and concomitant application of plasma rich in growth factor reported no cases of MRONJ and a survival implant rate of $98.7 \%$ (49). Successful dental implant installation has also been reported in patients using alendronate for 3 years who received antibiotic prophylaxis for 7 days before the procedure, with favorable osseointegration observed in $98 \%$ of the patients (50).

Despite the low risk of MRONJ with oral bisphosphonates, only a few studies have accurately defined the risk of developing MRONJ in the presence of dental implants. Approximately 20\% and $80 \%$ of the patients who developed MRONJ in the vicinity of dental implants have osteoporosis and oncologic disease, respectively (51). Although the risk of MRONJ is significantly higher in patients using intravenous bisphosphonates, an association between peri-implantitis and the etiology of MRONJ can also be observed in patients who use oral bisphosphonates $(48,51)$.

The use of minimally invasive techniques and soft tissue bone coverage have been described as factors that considerably reduce the incidence of complications after dental implant treatment (50). Measures such as avoiding bone augmentations and administration of antimicrobial prophylaxis are also recommended in these patients $(52)$.

Since infection around the implants represents a notable risk factor for MRONJ development, the implant presence itself - and not only the implant surgery - may trigger MRONJ $(3,43,46)$. In the informed consent form, patients should confirm that they are aware of possible long-term implant failure and the risk of MRONJ on implant sites (Figure 1) $(8,47)$.

\section{Endodontic, periodontal, and orthodontic treatment in patients with osteoporosis receiving antiresorptive therapy}

A controlled clinical trial on the risk of MRONJ related to conservative root canal treatment showed satisfactory outcomes such as periradicular healing in patients taking long-term oral bisphosphonates (53). 
Although periodontal disease may benefit from systemic bisphosphonate therapy (54), the disease itself is considered a risk factor for MRONJ (55). Anaerobic bacteria representative of periodontal microbiota were the main findings in the necrotic bone samples collected from MRONJ cases, suggesting that periodontal infection could actually initiate the process in patients undergoing antiresorptive treatment (56).

No studies have attributed orthodontic treatment to increased risk of MRONJ. While no clinical studies have been designed to specifically address orthodontic treatment in patients taking antiresorptive drugs for osteoporosis, one report has described three patients using oral bisphosphonates who, during orthodontic treatment, presented decreased tooth movement and difficulty for space closure and paralleling of roots (57).

Biologically, bisphosphonates can impact orthodontic treatment by impeding tooth movement due to osteoclast destruction and decreased microcirculation, limiting bone turnover and remodeling (58). Patients receiving bisphosphonate therapy must be aware of difficulties in closing the extraction spaces and paralleling the roots (Figure 1) (47).

\section{MRONJ management in patients with osteoporosis}

The AAOMS position paper recommends nonsurgical local treatment as the therapy of choice for earlystage MRONJ (8). However, surgical treatment is recommended for lesions refractory to initial treatment and those at an advanced stage. Bone sequestrations that are a constant source of soft tissue irritation and loose bony sequestra should be removed or recontoured so that soft tissue healing can be optimized (8).

In a study using the AAOMS protocol to treat patients with MRONJ, cure was achieved in $93 \%$ of the cases (59). However, only $34 \%$ of the sample showed improvement with nonsurgical treatment alone. Surgical procedures were performed in $62 \%$ of the patients and were effective in $94 \%$ of the sequestrectomies and $100 \%$ of the resections. In another study, conservative or surgical treatment was effective in $72 \%$ of the patients (60). Conservative treatment was refractory in $44 \%$ of the lesions, while surgical treatment was effective in all cases.

Surgical procedure can be used as the first line of treatment or in lesions refractory to conservative treatment $(59,61)$. However, surgeries under intravenous sedation or general anesthesia have shown better results, and patients with MRONJ could benefit from treatments more invasive than bone curettage performed under local anesthesia (61). The surgical treatment associated with the use of platelet-rich fibrin has also been effective, but it is not possible to ascertain if the cure was caused by the surgical procedure or by adjuvant biological materials (62). Recent studies have proposed surgeries to be performed even in the early stages of the disease, with a better prognosis (24).

Reports related to the medical treatment of MRONJ available in the literature are mostly case reports or small case series. The results of these studies are generally favorable to the use of teriparatide, although no definite answer for this approach is available due to the small number of patients treated and the lack of a control group in most reports $(63,64)$. These studies show improvement in serum markers or bone regeneration ratio, but do not show resolution of MRONJ.

In contrast to these previous series, a study that compared the local application of recombinant human bone morphogenetic protein-2 (rhBMP-2) alone or combined with short-term teriparatide versus no treatment (control group) observed that patients who received rhBMP-2 plus teriparatide had better outcomes than those who received rhBMP-2 alone or controls, suggesting that the administration of rhBMP-2 may maximize bone regeneration after surgery (63). More studies are still needed to recommend pharmacological treatment for the management of MRONJ.

In conclusion, antiresorptive medications are the most used pharmacological treatment for osteoporosis and reduce the risk of all types of fragility fractures. Although only scarce evidence links the development of MRONJ to the use of antiresorptive agents in the context of osteoporosis, these agents are considered a risk factor for MRONJ. Despite rare reports of MRONJ in patients with osteoporosis taking antiresorptive drugs, the severity of the impact of MRONJ in patients' quality of life makes it imperative for appropriate diagnosis, prevention, and treatment of this complication. Health care professionals should always work together to improve patients' safety and attain better outcomes.

Disclosure: no potential conflict of interest relevant to this article was reported.

\section{REFERENCES}

1. AghalooTL, Felsenfeld AL, Tetradis S. Osteonecrosis of the jaw in a patient on Denosumab. J Oral Maxillofac Surg. 2010;68(5):959-63. 
2. Estilo CL, Fornier M, Farooki A, Carlson D, Bohle G 3rd, Huryn JM. Osteonecrosis of the jaw related to bevacizumab. J Clin Oncol. 2008;26(24):4037-8.

3. Radominski SC, Bernardo W, Paula AP, Albergaria BH, Moreira C, Fernandes $\mathrm{CE}$, et al. Brazilian guidelines for the diagnosis and treatment of postmenopausal osteoporosis. Rev Bras Reumatol. 2017;57(Suppl 2):452-66.

4. Ruggiero SL, Mehrotra B, RosenbergTJ, Engroff SL. Osteonecrosis of the jaws associated with the use of bisphosphonates: a review of 63 cases. J Oral Maxillofac Surg. 2004;62(5):527-34.

5. Drake MT, Clarke BL, Khosla S. Bisphosphonates: mechanism of action and role in clinical practice. Mayo Clin Proc. 2008;83(9):1032-45.

6. Pinheiro MM, Ciconelli RM, Martini LA, Ferraz MB. Clinical risk factors for osteoporotic fractures in Brazilian women and men: the Brazilian Osteoporosis Study (BRAZOS). Osteoporos Int. 2009;20(3):399-408.

7. Aziziyeh R, Amin M, Habib M, Garcia Perlaza J, Szafranski K, McTavish RK, et al. The burden of osteoporosis in four Latin American countries: Brazil, Mexico, Colombia, and Argentina. J Med Econ. 2019;22(7):638-44.

8. Ruggiero SL, Dodson TB, Fantasia J, Goodday R, Aghaloo T, Mehrotra B, et al. American Association of Oral and Maxillofacial Surgeons position paper on medication-related osteonecrosis of the jaw - 2014 update. J Oral Maxillofac Surg. 2014;72(10):1938-56.

9. Maeda SS, Lazaretti-Castro M. An overview on the treatment of postmenopausal osteoporosis. Arq Bras Endocrinol Metabol. 2014;58(2):162-71.

10. Marx RE. Pamidronate (Aredia) and zoledronate (Zometa) induced avascular necrosis of the jaws: a growing epidemic. J Oral Maxillofac Surg. 2003;61(9):1115-7.

11. Odvina CV, Zerwekh JE, Rao DS, Maalouf N, Gottschalk FA, Pak CY. Severely suppressed bone turnover: a potential complication of alendronate therapy. J Clin Endocrinol Metab. 2005;90(3):1294-301.

12. Ott SM. What is the optimal duration of bisphosphonate therapy? Cleve Clin J Med. 2011;78(9):619-30.

13. Bone HG, Wagman RB, Brandi ML, Brown JP, Chapurlat $R$, Cummings SR, et al. 10 years of denosumab treatment in postmenopausal women with osteoporosis: results from the phase 3 randomised randomized FREEDOM trial and open-label extension. Lancet Diabetes Endocrinol. 2017;5(7):513-23.

14. Tsourdi E, Langdahl B, Cohen-Solal M, Aubry-Rozier B, Eriksen EF, Guanabens N, et al. Discontinuation of Denosumab therapy for osteoporosis: A systematic review and position statement by ECTS. Bone. 2017;105:11-7.

15. Khan AA, Morrison A, Hanley DA, Felsenberg D, McCauley LK, O'Ryan F, et al. Diagnosis and management of osteonecrosis of the jaw: a systematic review and international consensus. J Bone Miner Res. 2015;30(1):3-23.

16. Japanese Allied Committee on Osteonecrosis of the Jaw, Yoneda T, Hagino H, Sugimoto T, Ohta H, Takahashi S, Soen S, et al. Antiresorptive agent-related osteonecrosis of the jaw: Position Paper 2017 of the Japanese Allied Committee on Osteonecrosis of the Jaw. J Bone Miner Metab. 2017;35(1):6-19.

17. Khan AA, Morrison A, Kendler DL, Rizzoli R, Hanley DA, Felsenberg $D$, et al. Case-Based Review of Osteonecrosis of the Jaw (ONJ) and Application of the International Recommendations for Management From the International Task Force on ONJ. J Clin Densitom. 2017;20(1):8-24.

18. Soares AL, Simon S, Gebrim LH, Nazario ACP, Lazaretti-Castro M. Prevalence and risk factors of medication-related osteonecrosis of the jaw in osteoporotic and breast cancer patients: a crosssectional study. Support Care Cancer. 2020;28(5):2265-71.
19. Borromeo GL, Brand C, Clement JG, McCullough M, Crighton L, Hepworth G, et al. A large case-control study reveals a positive association between bisphosphonate use and delayed dental healing and osteonecrosis of the jaw. J Bone Miner Res. 2014;29(6):1363-8.

20. Eiken PA, Prieto-Alhambra D, Eastell R, Abrahamsen B. Surgically treated osteonecrosis and osteomyelitis of the jaw and oral cavity in patients highly adherent to alendronate treatment: a nationwide user-only cohort study including over 60,000 alendronate users. Osteoporos Int. 2017;28(10):2921-8.

21. Zavras Al, Shanmugham JR. Bisphosphonates, Osteoporosis, and Osteonecrosis of the Jaw: A Critical Review of a Large Nested Case-Control Study. J Evid Based Dent Pract. 2016;16(2):136-8.

22. Jeong HG, Hwang JJ, Lee JH, Kim YH, Na JY, Han SS. Risk factors of osteonecrosis of the jaw after tooth extraction in osteoporotic patients on oral bisphosphonates. Imaging Sci Dent. 2017;47(1):45-50.

23. Yamazaki T, Yamori M, Ishizaki T, Asai K, Goto K, Takahashi K, et al. Increased incidence of osteonecrosis of the jaw after tooth extraction in patients treated with bisphosphonates: a cohort study. Int J Oral Maxillofac Surg. 2012;41(11):1397-403.

24. Schiodt M, Otto S, Fedele S, Bedogni A, Nicolatou-Galitis O, Guggenberger R, et al. Workshop of European task force on medication-related osteonecrosis of the jaw-Current challenges. Oral Dis. 2019;25(7):1815-21.

25. Gaudin E, Seidel L, Bacevic M, Rompen E, Lambert F. Occurrence and risk indicators of medication-related osteonecrosis of the jaw after dental extraction: a systematic review and meta-analysis. $\mathrm{J}$ Clin Periodontol. 2015;42(10):922-32.

26. Otto S, Troltzsch M, Jambrovic V, Panya S, Probst F, Ristow O, et al. Tooth extraction in patients receiving oral or intravenous bisphosphonate administration: A trigger for BRONJ development? J Craniomaxillofac Surg. 2015;43(6):847-54.

27. Adler RA, El-Hajj Fuleihan G, Bauer DC, Camacho PM, Clarke BL, Clines GA, et al. Managing Osteoporosis in Patients on LongTerm Bisphosphonate Treatment: Report of a Task Force of the American Society for Bone and Mineral Research. J Bone Miner Res. 2016;31(1):16-35.

28. Jung SY, Suh HS, Park JW, Kwon JW. Drug holiday patterns and bisphosphonate-related osteonecrosis of the jaw. Oral Dis. 2019;25(2):471-80.

29. McClung M, Harris ST, Miller PD, Bauer DC, Davison KS, Dian L, et al. Bisphosphonate therapy for osteoporosis: benefits, risks, and drug holiday. Am J Med. 2013;126(1):13-20.

30. Anagnostis P, Paschou SA, Mintziori G, Ceausu I, Depypere H, Lambrinoudaki I, et al. Drug holidays from bisphosphonates and denosumab in postmenopausal osteoporosis: EMAS position statement. Maturitas. 2017;101:23-30.

31. Hasegawa $T$, Kawakita A, Ueda N, Funahara R, Tachibana A, Kobayashi $M$, et al. A multicenter retrospective study of the risk factors associated with medication-related osteonecrosis of the jaw after tooth extraction in patients receiving oral bisphosphonate therapy: can primary wound closure and a drug holiday really prevent MRONJ? Osteoporos Int. 2017;28(8):2465-73.

32. Hayashida S, Yanamoto S, Fujita S, Hasegawa T, Komori T, Kojima $Y$, et al. Drug holiday clinical relevance verification for antiresorptive agents in medication-related osteonecrosis cases of the jaw. J Bone Miner Metab. 2020;38(1):126-34.

33. Marx RE, Cillo JE Jr, Ulloa JJ. Oral bisphosphonate-induced osteonecrosis: risk factors, prediction of risk using serum CTX testing, prevention, and treatment. J Oral Maxillofac Surg. 2007;65(12):2397-410.

34. Hutcheson A, Cheng A, Kunchar R, Stein B, Sambrook P, Goss A. A C-terminal crosslinking telopeptide test-based protocol 
for patients on oral bisphosphonates requiring extraction: a prospective single-center controlled study. J Oral Maxillofac Surg. 2014;72(8):1456-62.

35. Kunchur R, Need A, Hughes T, Goss A. Clinical investigation of C-terminal cross-linking telopeptide test in prevention and management of bisphosphonate-associated osteonecrosis of the jaws. J Oral Maxillofac Surg. 2009;67(6):1167-73.

36. Salgueiro M, Stribos M, Zhang LF, Stevens M, Awad ME, Elsalanty M. Value of preoperative CTX serum levels in the prediction of medication-related osteonecrosis of the jaw (MRONJ): a retrospective clinical study. EPMA J. 2019;10(1):21-9.

37. Awad ME, Sun C, Jernigan J, Elsalanty M. Serum C-terminal cross-linking telopeptide level as a predictive biomarker of osteonecrosis after dentoalveolar surgery in patients receiving bisphosphonate therapy: Systematic review and meta-analysis. J Am Dent Assoc. 2019;150(8):664-75.e8.

38. Lorenzo-Pouso Al, Perez-Sayans M, Gonzalez-Palanca S, Chamorro-Petronacci C, Bagan J, Garcia-Garcia A. Biomarkers to predict the onset of biphosphonate-related osteonecrosis of the jaw: A systematic review. Med Oral Patol Oral Cir Bucal. 2019;24(1):e26-e36.

39. Koo $\mathrm{CH}$, Lee JH. Evaluation of mandibular cortical bone ratio on computed tomography images in patients taking bisphosphonates. Maxillofac Plast Reconstr Surg. 2018;40(1):17.

40. Torres SR, Chen CS, Leroux BG, Lee PP, Hollender LG, Lloid M, et al. Mandibular inferior cortical bone thickness on panoramic radiographs in patients using bisphosphonates. Oral Surg Oral Med Oral Pathol Oral Radiol. 2015;119(5):584-92.

41. Barngkgei I, Halboub E, Almashraqi A. Effect of bisphosphonate treatment on the jawbone: an exploratory study using periapical and panoramic radiographic evaluation. Oral Radiol. 2019;35(2):159-70.

42. Diniz-Freitas $M$, Fernandez-Montenegro $P$, Fernandez-Feijoo J, Limeres-Posse J, Gonzalez-Mosquera A, Vazquez-Garcia E, et al. Mandibular cortical indices on cone-beam computed tomography images in osteoporotic women on treatment with oral bisphosphonates. Gerodontology. 2016;33(2):155-60.

43. Kubo R, Ariji Y, Taniguchi T, Nozawa M, Katsumata A, Ariji E. Panoramic radiographic features that predict the development of bisphosphonate-related osteonecrosis of the jaw. Oral Radiol. 2018;34(2):151-60

44. Diniz-Freitas M, Limeres J. Prevention of medication-related osteonecrosis of the jaws secondary to tooth extractions. A systematic review. Med Oral Patol Oral Cir Bucal. 2016;21(2):e250-9.

45. Bermudez-Bejarano EB, Serrera-Figallo MA, Gutierrez-Corrales A, Romero-Ruiz MM, Castillo-de-Oyague R, Gutierrez-Perez JL, et al. Prophylaxis and antibiotic therapy in management protocols of patients treated with oral and intravenous bisphosphonates. J Clin Exp Dent. 2017;9(1):e141-e9.

46. Mozzati M, Arata V, Gallesio G. Tooth extraction in patients on zoledronic acid therapy. Oral Oncol. 2012;48(9):817-21.

47. Cavalcanti EFF, Gomes AOF, Torres SR. Osteonecrosis of the Jaws: The Awareness of the Patient at Risk. Biomed J Sci Tech Res 2017;1(4):859-60.

48. Giovannacci I, Meleti M, Manfredi M, Mortellaro C, Greco Lucchina A, Bonanini M, et al. Medication-Related Osteonecrosis of the Jaw Around Dental Implants: Implant Surgery-Triggered or Implant Presence-Triggered Osteonecrosis? J Craniofac Surg. 2016;27(3):697-701.

49. Mozzati M, Arata V, Giacomello M, Del Fabbro M, Gallesio G, Mortellaro $\mathrm{C}$, et al. Failure risk estimates after dental implants placement associated with plasma rich in growth factor-Endoret in osteoporotic women under bisphosphonate therapy. J Craniofac Surg. 2015;26(3):749-55.

50. Tallarico M, Canullo L, Xhanari E, Meloni SM. Dental implants treatment outcomes in patient under active therapy with alendronate: 3-year follow-up results of a multicenter prospective observational study. Clin Oral Implants Res. 2016;27(8):943-9.

51. Troeltzsch M, Cagna D, Stahler P, Probst F, Kaeppler G, Troeltzsch $M$, et al. Clinical features of peri-implant medication-related osteonecrosis of the jaw: Is there an association to periimplantitis? J Craniomaxillofac Surg. 2016;44(12):1945-51.

52. Walter C, Al-Nawas B, Wolff T, Schiegnitz E, Grotz KA. Dental implants in patients treated with antiresorptive medication - a systematic literature review. Int J Implant Dent. 2016;2(1):9.

53. Hsiao A, Glickman G, He J. A retrospective clinical and radiographic study on healing of periradicular lesions in patients taking oral bisphosphonates. J Endod. 2009;35(11):1525-8.

54. Penoni DC, Torres SR, Farias ML, Fernandes TM, Luiz RR, Leao AT. Association of osteoporosis and bone medication with the periodontal condition in elderly women. Osteoporos Int. 2016;27(5):1887-96.

55. Nicolatou-Galitis O, Schiodt M, Mendes RA, Ripamonti C, Hope $S$, Drudge-Coates L, et al. Medication-related osteonecrosis of the jaw: definition and best practice for prevention, diagnosis, and treatment. Oral Surg Oral Med Oral Pathol Oral Radiol. 2019;127(2):117-35.

56. Hallmer F, Bjornland T, Nicklasson A, Becktor JP, Andersson G. Osteonecrosis of the jaw in patients treated with oral and intravenous bisphosphonates: experience in Sweden. Oral Surg Oral Med Oral Pathol Oral Radiol. 2014;118(2):202-8.

57. Zahrowski JJ. Optimizing orthodontic treatment in patients taking bisphosphonates for osteoporosis. Am J Orthod Dentofacial Orthop. 2009;135(3):361-74.

58. Rinchuse DJ, Rinchuse DJ, Sosovicka MF, Robison JM, Pendleton R. Orthodontic treatment of patients using bisphosphonates: a report of 2 cases. Am J Orthod Dentofacial Orthop. 2007;131(3):321-6.

59. Shintani T, Hayashido $Y$, Mukasa H, Akagi E, Hoshino M, Ishida $Y$, et al. Comparison of the prognosis of bisphosphonaterelated osteonecrosis of the jaw caused by oral and intravenous bisphosphonates. Int J Oral Maxillofac Surg. 2015;44(7):840-4.

60. Mercer E, Norton T, Woo S, Treister N, Dodson TB, Solomon DH. Ninety-one osteoporosis patients affected with bisphosphonaterelated osteonecrosis of the jaw: a case series. Calcif Tissue Int. 2013;93(3):241-8.

61. Nisi M, La Ferla F, Karapetsa D, Gennai S, Ramaglia L, Graziani $\mathrm{F}$, et al. Conservative surgical management of patients with bisphosphonate-related osteonecrosis of the jaws: a series of 120 patients. Br J Oral Maxillofac Surg. 2016;54(8):930-5.

62. Mourao FABC, Calasans-Maia MD, Del Fabbro M, Le Drapper Vieira F, Coutinho de Mello Machado R, Capella R, et al. The use of Platelet-rich Fibrin in the management of medicationrelated osteonecrosis of the jaw: A case series. J Stomatol Oral Maxillofac Surg. 2020;121(1):84-9.

63. Jung J, Yoo HY, Kim GT, Lee JW, LeeYA, Kim DY, et al. Short-Term Teriparatide and Recombinant Human Bone Morphogenetic Protein-2 for Regenerative Approach to Medication-Related Osteonecrosis of the Jaw: A Preliminary Study. J Bone Miner Res. 2017;32(12):2445-52.

64. Kakehashi $H$, Ando $T$, Minamizato $T$, Nakatani $Y$, Kawasaki T, Ikeda $\mathrm{H}$, et al. Administration of teriparatide improves the symptoms of advanced bisphosphonate-related osteonecrosis of the jaw: preliminary findings. Int J Oral Maxillofac Surg. 2015;44(12):1558-64. 